\title{
Confecção e Análise de um Piranômetro Fotovoltaico de Baixo Custo
}

\author{
João Frederico Souza de Paula ${ }^{[1]}$, George Bezerra Ribeiro ${ }^{[2]}$, Saulo Tasso Araújo da Silva ${ }^{[3]}$ e Vladimir \\ Batista Figueirêdo ${ }^{[4]}$ \\ ${ }^{[1]}$ Universidade Federal Rural do Semi-árido; derico@ motoeste.com.br \\ ${ }^{[2]}$ Universidade Federal Rural do Semi-árido; george @ ufersa.edu.br \\ ${ }^{[3]}$ Universidade Federal Rural do Semi-árido; saulo@ufersa.edu.br \\ ${ }^{[4]}$ Universidade Federal Rural do Semi-árido; vladimir@ ufersa.edu.br \\ Recebido: 08/07/2020; \\ Aceito: $31 / 07 / 2020$ \\ Publicado: 11/09/2020.
}

Resumo: Este trabalho teve como objetivo o desenvolvimento e avaliação de um piranômetro fotovoltaico de baixo custo, construído desde materiais encontrados facilmente no mercado nacional. Para tanto, foram avaliados modelos de pequenos módulos fotovoltaicos de silício policristalino. Foi desenvolvido um protótipo de corpo de piranômetro com plástico de engenharia usinado. Também foram analisados os comportamentos de difusores construídos de três materiais diferentes, Thecnyl®, acrílico e PVC. Para o material escolhido foram testadas duas espessuras distintas e acabamento superficial polido e fosco. Todos os testes foram realizados em campo, na estação meteorológica da Universidade Federal Rural do Semi-Árido, em MossoróRN. Os resultados foram comparados com um piranômetro de classe secundária. Os dados dos experimentos foram coletados por um registrador automático e o seu tratamento foi realizado considerando as recomendações da norma ISO 9847:1992. O desempenho do modelo final do protótipo mostrou-se satisfatório para medições na escala horária e diária. Nas medições de valores totais horários, das 6 h00 às 18h00, o erro relativo percentual absoluto médio foi de $2,26 \%$, o mínimo de $0,433 \%$ e o máximo de $8,10 \%$. Para os valores totais diários, o erro relativo percentual absoluto médio foi de $0,50 \%$, o mínimo de $0,16 \%$ e o máximo de $0,93 \%$.

Palavras-chave: Radiação Solar; Sensor de Baixo Custo; Calibração.

Abstract: The present research aimed the development and the evaluation of a low cost photovoltaic pyranometer, built from easily found materials in the national market. Therefore, models of small polycrystalline silicon photovoltaic modules were evaluated. A pyranometer body prototype with was developed with machined engineering plastic. Also, the behavior of diffusers built with three different materials Thecnyl®, acrylic and polyvinyl chloride were analyzed. For the chosen one, two distinct thicknesses were tested, as well as polished and matte surface finishing. All the tests were performed in the field, in the Federal University Rural of Semiarid weather station, from Mossoró-RN. The results were compared to a secondary class reference pyranometer. The experiments data were collected by an automatic datalogger and its handling was accomplished considering the regulation ISO 9847:1992 recommendations. The performance of the prototype final model has shown itself satisfactory considering measurements in the hourly and daily scales. In the total hourly values measurements, since 6 a.m. to 6 p.m., the absolute percentage relative error was $2,26 \%$, the minimum was $0,433 \%$ and the maximum was $8,10 \%$. In the total daily values, the absolute percentage relative error was $0,50 \%$, the minimum was $0,16 \%$ and the maximum was $0,93 \%$.

Key-words: Solar radiation; Low cost sensor; Calibration. 


\section{INTRODUÇÃO}

$\mathrm{O}$ conhecimento da radiação solar que chega à superfície terrestre tem sido cada vez mais importante para subsidiar diversas atividades, como estudos meteorológicos, aproveitamento do recurso solar para fins energéticos, manejo e trato de culturas na agricultura irrigada, projetos de edificações, entre outras aplicações. Especialmente no que se refere ao aproveitamento para fins energéticos, o Brasil e, particularmente a região Nordeste, apresentam um grande potencial, que apenas recentemente começou a ser explorado, em razão da adoção de políticas públicas de incentivo ao uso da energia fotovoltaica.

O instrumento comumente utilizado para a medição da radiação solar costuma ser o piranômetro. No Brasil, esses instrumentos geralmente são importados, possuem ótima precisão e alto custo, o que torna o acesso a eles viável quase que exclusivamente às universidades, centros de pesquisa e empresas de grande porte. Devido a isso, na literatura encontram-se vários trabalhos [1-3] que realizaram pesquisas sobre o desenvolvimento de sensores de radiação solar, visando o melhor custo benefício, comparando-os com sensores comerciais de alta qualidade, conforme a ISO 9060:1990. Este cenário motiva e justifica o desenvolvimento de um modelo de piranômetro fotovoltaico com boa acurácia e baixo custo, que possibilite a disseminação do acesso a este dispositivo.

Desta forma, a pesquisa realizada no presente trabalho tem como objetivo a construção e análise das características de desempenho de um piranômetro do tipo fotovoltaico, comparando-o com um sensor de referência de classe secundária, utilizado normalmente como referência para calibração

\section{FUNDAMENTAÇÃO TEÓRICA}

Ao atravessar a atmosfera terrestre, a radiação solar sofre modificações nos seus comprimentos de onda e na orientação, devido ao processo de absorção, refração e reflexão, separando-a em dois tipos: direta e difusa. O piranômetro mede a soma de ambas as componentes, denominada radiação global.

Os elementos sensores utilizados nos piranômetros são, normalmente, termopilha ou célula fotovoltaica. O primeiro é constituído por um conjunto de termopares, que constitui o chamado termopilha a qual é posicionada sob um difusor, contendo um lado posicionado em uma superfície enegrecida voltada para o Sol e o outro voltado para o interior do instrumento ou sob uma superfície de cor clara. A diferença de temperatura nas juntas dos termopares expostos em condições diferentes à radiação solar produz uma tensão elétrica proporcional à radiação incidente [4]. O segundo tipo normalmente utiliza uma pequena célula fotovoltaica de silício como sensor, a qual é posicionada sob um difusor e exposta à radiação solar. Pelo efeito fotovoltaico, a célula produz uma corrente elétrica que, sob determinadas condições, é proporcional à intensidade da radiação incidente [4]. Isto ocorre quando a célula opera na região da curva de corrente versus tensão entre o ponto de operação em curto-circuito e um ponto próximo ao de máxima potência, onde a corrente passa a decrescer com o aumento da tensão. A operação nessa região de linearidade pode ser conseguida conectando a célula a uma resistência de valor suficientemente baixo. Um piranômetro com sensor fotovoltaico costuma ter resposta mais rápida e linear e um custo menor, em comparação com o que utiliza a termopilha [5]. A grande desvantagem do sensor fotovoltaico é sua reduzida faixa espectral de resposta, situada entre 400 e $1.100 \mathrm{~nm}$, inferior ao sensor de termopilha, que responde, normalmente, à faixa entre 285 e $2.800 \mathrm{~nm}$ [6].

\section{MATERIAIS E MÉTODOS}

O desenvolvimento do projeto do piranômetro fotovoltaico ocorreu em três etapas. Na primeira, foram escolhidos módulos fotovoltaicos de tamanho reduzido, de fácil acesso no mercado nacional e de baixo custo. 
Esses módulos foram testados em campo, para a determinação das suas características elétricas, levantamento da relação entre a radiação solar global e a corrente elétrica produzida e definição da resistência de carga que resultasse em um bom comportamento do conjunto, considerando a linearidade da resposta e a sensibilidade. Posteriormente, foi desenvolvido um projeto do corpo do piranômetro, sendo construídas três unidades, e realizados novos testes de campo para a escolha do material e características físicas do difusor. Os protótipos foram instalados no parque de sensores da Estação Meteorológica Automática (EMA) da Universidade Federal Rural do Semi-Árido - UFERSA ( $05^{\circ} 12^{\prime} 49^{\prime}$ ' S, $37^{\circ} 18^{\prime} 44^{\prime}$ W, $\left.38 \mathrm{~m}\right)$ e os experimentos foram realizados entre 3 de agosto de 2017 e 11 de março de 2018. Os dados dos testes de campo foram coletados por um registrador de dados do tipo datalogger, modelo Campbell Scientific, Inc. CR 3000. Nos testes, além das tensões dos protótipos de piranômetros fotovoltaicos, foi monitorada a radiação solar global, através de um piranômetro KIPP\&ZONEN CMP22 [7], utilizado neste estudo como referência. Este piranômetro de referência possui sensor do tipo termopilha, faixa espectral de 200 a $3600 \mathrm{~nm}$ e classificação de padrão secundário, de acordo com a norma ISO 9060:1990, tendo, portanto, o mais alto nível de acurácia para este tipo de instrumento [7].

O tratamento dos dados coletados e os procedimentos de calibração dos protótipos foram fundamentados na ISO 9847:1992, que descreve uma metodologia para calibração de piranômetros em campo usando um piranômetro de referência. Como a metodologia apresentada na ISO 9847:1992 é essencialmente aplicável a medições realizadas de forma manual, em séries de medições distintas para condições diferentes de céu, considerando céu estável sem nuvens, céu instável com algumas nuvens e céu nublado, foram feitas adaptações para o caso concreto: Em todos os testes realizados em campo, o datalogger foi programado para realizar leituras a cada 5 segundos e registrar as médias a cada 10 minutos, durante todo o período dos experimentos. Com isso, houve o aumento significativo do número de dados coletados em relação aos procedimentos especificados na norma.

\subsection{Ensaio e Seleção do Módulo Fotovoltaico}

A primeira etapa do desenvolvimento do protótipo foi o teste de módulos fotovoltaicos visando à escolha do modelo a ser adotado como elemento sensor da radiação solar global. Foram submetidos ao teste pequenos módulos fotovoltaicos policristalinos de dois modelos, um medindo 3 x $3 \mathrm{~cm}$ e a corrente e tensões nominais de $80 \mathrm{~mA}$ e $1 \mathrm{~V}$ (Figura 1, módulos 4, 5 e 6) e o outro medindo 5 x $5 \mathrm{~cm}$ com $160 \mathrm{~mA}$ de corrente e $2 \mathrm{~V}$ de tensão nominal (Figura 1, módulos 1, 2 e 3). Os módulos foram conectados a resistores de valores baixos o bastante para garantir a operação na região linear horizontal da curva I x V.

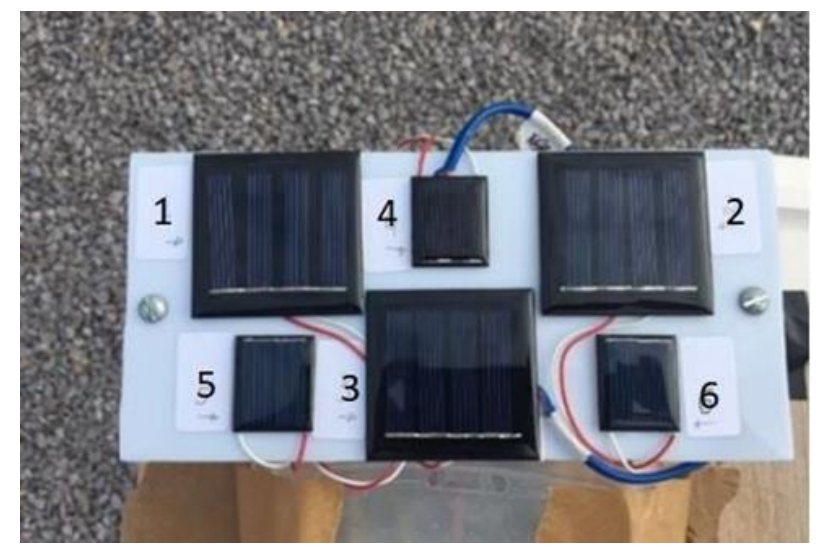

FIGURA 1. Sensores em teste. (Autoria Própria)

\subsection{Projeto e Construção do Corpo do Sensor}


A segunda etapa do desenvolvimento do protótipo foi o projeto de um corpo para o sensor, que fosse compatível com as dimensões do módulo fotovoltaico escolhido e que contemplasse a inserção de um difusor. Tomou-se como referência o formato cilíndrico, vastamente adotado em piranômetros que utilizam fotodiodos como sensor. O corpo foi confeccionado em material plástico tipo THECNYL ${ }^{\circledR 1}$, fixado em uma base confeccionada por um flange de PVC. No centro da estrutura do corpo foi feito um rebaixamento, onde foi inserido o módulo fotovoltaico conectado a um cabo tipo manga de duas vias com diâmetro de 26 AWG $(0,4049$ $\mathrm{mm})$. Foram confeccionadas tampas, também de THECNYL ${ }^{\circledR}$, sendo um modelo fechado no topo e outro vazado para inserção de difusores de outros materiais. A Figura 2 mostra uma imagem do protótipo montado com difusor de acrílico.

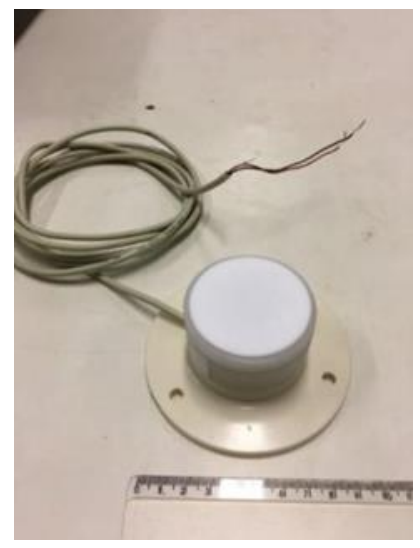

FIGURA 2. Corpo do piranômetro proposto com difusor de acrílico. (Autoria própria)

\subsection{Definição do Difusor}

A última etapa do processo de desenvolvimento do piranômetro de baixo custo foi a identificação de um difusor que apresentasse bons resultados, selecionando-se entre vários tipos de material, espessura e acabamento da superfície. Em um piranômetro fotovoltaico, o difusor possui a função de distribuir uniformemente e, também, de reduzir a intensidade de radiação incidente no módulo fotovoltaico, aumentando sua vida útil. Em alguns projetos, verifica-se que ele é confeccionado em um formato especial, geralmente cônico ou semiesférico, visando reduzir o erro de cosseno do instrumento. Neste projeto não houve este objetivo, uma vez que a construção de difusores nestes formatos costumam encarecer a sua fabricação. Assim, foram testados difusores apenas no formato cilíndrico, com materiais, espessuras e superfícies variadas. Este formato geométrico é de fácil construção, tendo sido utilizado em trabalhos de outros autores, com resultados satisfatórios [1]. Foram testados difusores de THECNYL ${ }^{\circledR}$, PVC [2] e acrílico [1], materiais de baixo custo e fácil aquisição no mercado local.

Nesta etapa, foram realizados três ciclos de testes, utilizando-se três protótipos do piranômetro, juntamente com o modelo de referência - KIPP\&ZONEN CMP22, todos conectados ao registrador de dados. Em cada ciclo de testes, considerando a metodologia de tratamento dos dados recomendada pela ISO 9847, os valores médios de cada 10 minutos obtidos por cada protótipo para ângulos de elevação do Sol superiores a $20^{\circ}$, foram comparados com os valores do piranômetro de referência, conforme definido na Equação 1, e calculada a sensibilidade de cada protótipo.

$$
\frac{\sum_{t=1}^{n}\left|\frac{y t-\hat{\boldsymbol{y}} t}{y t}\right|}{n}
$$

Onde n é o número de dados, y é o dado proveniente do protótipo e ŷ é o dado do piranômetro de referência. 
Em seguida, foram desconsideradas todas as leituras que apresentavam discrepância superior a $2 \%$ e calculada novamente a sensibilidade média de cada protótipo. Além da Equação 1, foram usados os índices estatísticos "coeficiente de determinação $\left(\mathrm{R}^{2}\right)$ ", "coeficiente de variação $(\mathrm{CV})$ " e os "erros relativos absolutos", para efeitos de comparação dos modelos.

\subsubsection{Definição do Material do Difusor}

O primeiro ciclo de testes dos difusores teve o objetivo de selecionar o material mais adequado. Durante três dias foram testados, simultaneamente, três materiais para a composição do difusor, os quais foram: $\mathrm{O}$ THECNYL ${ }^{\circledR}$, o acrílico leitoso e o PVC, com espessuras de $4 \mathrm{~mm}, 3 \mathrm{~mm}$ e 1,5 mm, respectivamente. Em relação ao THECNYL ${ }^{\circledR}$, não foi encontrada na literatura referências sobre sua aplicação como difusor para radiação solar, mas as características de fácil disponibilidade, baixo custo e sua propriedade de resistência a altas temperaturas, despertou o interesse de incluí-lo na comparação com os outros dois materiais.

\subsubsection{Espessura e Acabamento da Superfície do Difusor}

O segundo ciclo de testes teve como objetivo verificar o efeito da espessura do difusor na resposta relativa do piranômetro, em comparação com a referência. Durante três dias foram testados dois protótipos com os difusores compostos pelo material escolhido no primeiro ciclo de testes, mas com espessuras distintas e ambos com superfície polida. Por fim, no terceiro e último ciclo de testes, com duração de cinco dias, dois protótipos foram testados, um com superfície polida e o outro fosco, visando identificar os efeitos do acabamento superficial do difusor no comportamento do piranômetro.

\section{RESUltADOS}

\subsection{Módulos Fotovoltaicos}

Na Figura 3 pode ser visto o teste realizado em campo, entre 3 e 22 de agosto de 2017, no qual os módulos fotovoltaicos estavam conectados a resistores em paralelo. Observa-se também o piranômetro KIPP\&ZONEN CMP22 e o Datalogger, abrigado em caixa de proteção de fibra de vidro.

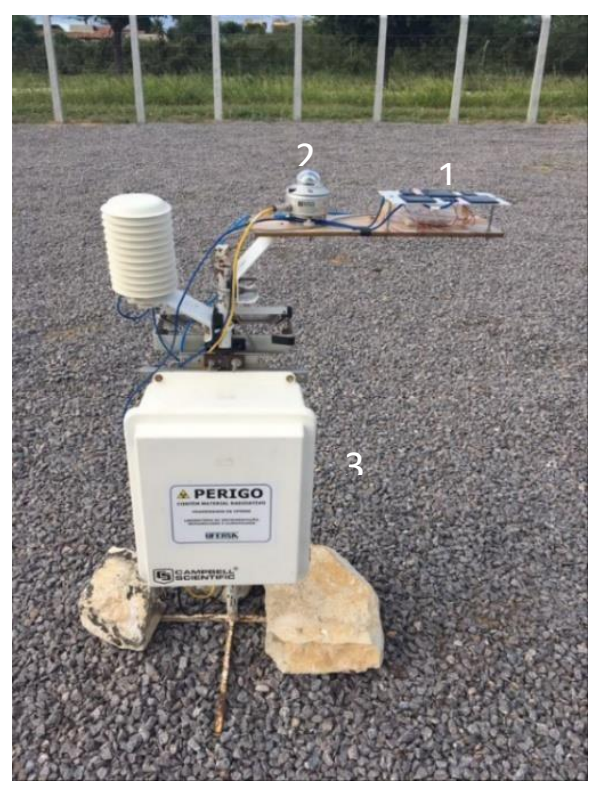

FIGURA 3. Teste dos elementos sensores. (Autoria própria) 
Nota: Os módulos em teste (1), o piranômetro de referência (2) e o Datalogger, situado no interior da caixa de fibra de vidro (3).

O teste com seis módulos fotovoltaicos conectados a resistências escolhidas de acordo com o item 3.1 mostrou que os sensores de menores dimensões apresentaram resultados satisfatórios, motivando a sua escolha para comporem os protótipos. A Tabela 1 mostra a Sensibilidade e o Coeficiente de determinação dos resultados destes módulos, em comparação com os dados do piranômetro de referência.

TABELA 1. Características de resposta dos módulos escolhidos. (Autoria própria)

\begin{tabular}{|c|c|c|}
\hline Módulo & Sensibilidade & Coeficiente de determinação $\left(R^{\mathbf{2}}\right)$ \\
\hline Módulo 1 & $1,696 \mathrm{~W} \cdot \mathrm{m}^{-2} / \mathrm{mV}$ & 0,999 \\
\hline Módulo 2 & $1,831 \mathrm{~W} \cdot \mathrm{m}^{-2} / \mathrm{mV}$ & 0,997 \\
\hline Módulo 3 & $1,780 \mathrm{~W} \cdot \mathrm{m}^{-2} / \mathrm{mV}$ & 0,996 \\
\hline
\end{tabular}

Os resultados da Tabela 1 foram obtidos com os módulos conectados em paralelo a resistências de 5,6 ohms, valor que apresentou boa relação Irradiação x Tensão. Todos os módulos apresentaram uma boa linearidade com os dados do piranômetro de referência, com coeficientes de determinação superiores a 0,99 . Também apresentaram uma boa sensibilidade, em média de $1,769 \mathrm{~W} . \mathrm{m}^{-2} / \mathrm{mV}$, o que os qualifica para o uso com dataloggers e até mesmo para leitura direta, com voltímetro.

\subsection{Avaliação dos Protótipos com Diferentes Difusores}

Os resultados dos experimentos de campo realizados para avaliação e escolha do material do difusor, sua espessura e seu acabamento superficial são apresentados nesta seção. Observa-se na Figura 4 o conjunto de protótipos de piranômetro, ao lado do piranômetro de referência, modelo KIPP\&ZONEN CMP22.

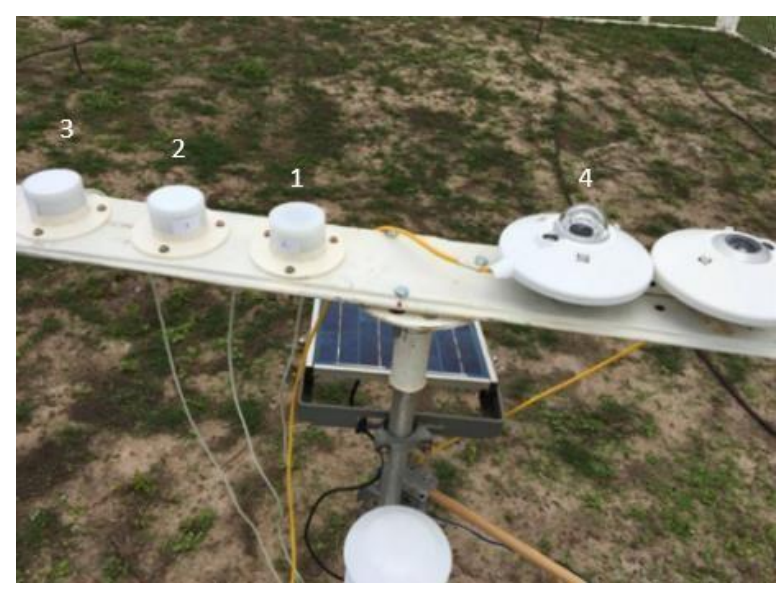

FIGURA 4. Protótipos de piranômetros com os difusores em teste e o piranômetro de referência,

KIPP\&ZONEN CMP22. (Autoria própria)

Nota: os protótipos estão enumerados de 1 a 3 e o piranômetro de referência com a enumeração 4.

\subsubsection{Material do Difusor}

Na Figura 5, verificam-se os valores dos sinais de saída dos protótipos, em milivolts, com os três materiais como difusor: THECNYL ${ }^{\circledR}$, acrílico e PVC. Também estão apresentados os valores medidos no piranômetro de referência, convertidos em W. $\mathrm{m}^{-2}$, no eixo vertical secundário. Os dados referem-se ao período de três dias, 
entre 23 e 25 de fevereiro de 2018, de 7h00min as 16h30min, período em que o ângulo de elevação do Sol encontrava-se acima de $20^{\circ}$. O céu neste intervalo apresentou-se parcialmente nublado e não houve a ocorrência de chuvas.

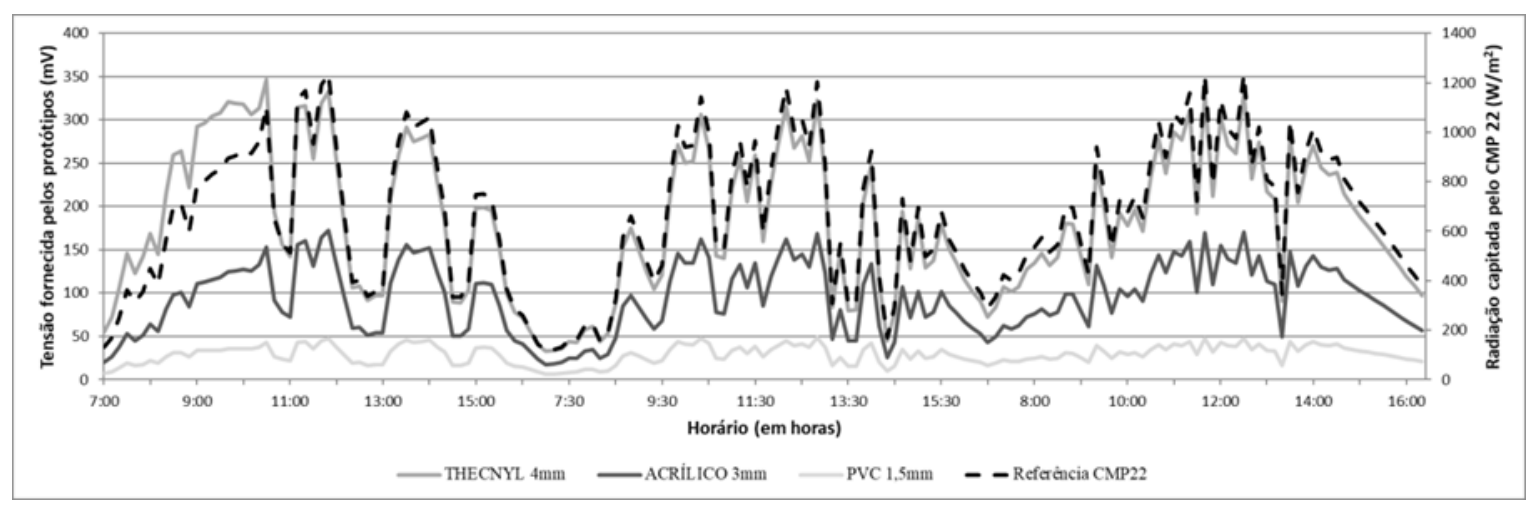

FIGURA 5. Resultados dos piranômetros protótipos com difusores de três materiais distintos e comparativamente ao piranômetro de referência. (Autoria própria)

O protótipo com difusor de THECNYL ${ }^{\circledR}$ apresentou uma sensibilidade variável, sendo maior no início do primeiro dia e menor no restante do período do experimento. Uma provável causa desta variação pode ter sido a ocorrência de chuva no local durante a noite anterior, fazendo com que o THECNYL ${ }^{\circledR}$ absorvesse umidade, alterando a sua translucidez e, consequentemente, aumentando também a sensibilidade do piranômetro. Em relação aos outros dois materiais, não ocorreu alteração porque as suas superfícies não possuem a propriedade de absorção como o primeiro material. A Tabela 2 traz as especificações e resultados para cada piranômetro com os difusores avaliados.

TABELA 2. Respostas dos piranômetros com difusores de três materiais diferentes. (Autoria própria)

\begin{tabular}{|c|c|c|c|c|c|}
\hline Protótipo & Material do Difusor & Espessura do Difusor & Sensibilidade & Coeficiente de & Variação \\
\hline 1 & THECNYL $^{\circledR}$ & $4 \mathrm{~mm}$ & $3,646 \mathrm{~W} \cdot \mathrm{m}^{-2} / \mathrm{mV}$ & $10,2 \%$ & 0,931 \\
\hline 2 & Acrílico & $3 \mathrm{~mm}$ & $6,958 \mathrm{~W} \cdot \mathrm{m}^{-2} / \mathrm{mV}$ & $3,5 \%$ & 0,996 \\
\hline 3 & PVC & $1,5 \mathrm{~mm}$ & $22,071 \mathrm{~W} \cdot \mathrm{m}^{-2} / \mathrm{mV}$ & $11,8 \%$ & 0,943 \\
\hline
\end{tabular}

Dentre os protótipos avaliados, a unidade com difusor de acrílico destacou-se, apresentando uma linearidade melhor e um coeficiente de variação menor. O THECNYL ${ }^{\circledR}$ apresentou uma sensibilidade melhor, mas um elevado Coeficiente de Variação (CV), em comparação com o acrílico. O PVC obteve os piores resultados em relação ao $\mathrm{CV}$ e sensibilidade, comparado aos demais. Logo, o acrílico foi escolhido como material do difusor.

\subsubsection{Avaliação de Diferentes Espessuras e Acabamento Superficial do Difusor}

Após a escolha do acrílico como o material para confecção do difusor, foram avaliadas amostras de duas espessuras, $3 \mathrm{~mm}$ ou 1,5 mm, e verificadas suas respostas à irradiação solar, comparativamente ao piranômetro de referência. O experimento foi realizado no período de três dias, entre 27 de fevereiro e 01 de março de 2018 , das 7h00min às $16 \mathrm{~h} 30 \mathrm{~min}$, e foram considerados os valores para ângulos de elevação do Sol superiores a $20^{\circ}$. O céu, neste intervalo, apresentou-se claro e com pouca presença de nuvens no primeiro dia, mas nos dias 
seguintes a nebulosidade aumentou gradativamente até que, no terceiro dia, o tempo estava nublado. A Figura 6 traz o registro do comportamento dos dois protótipos.

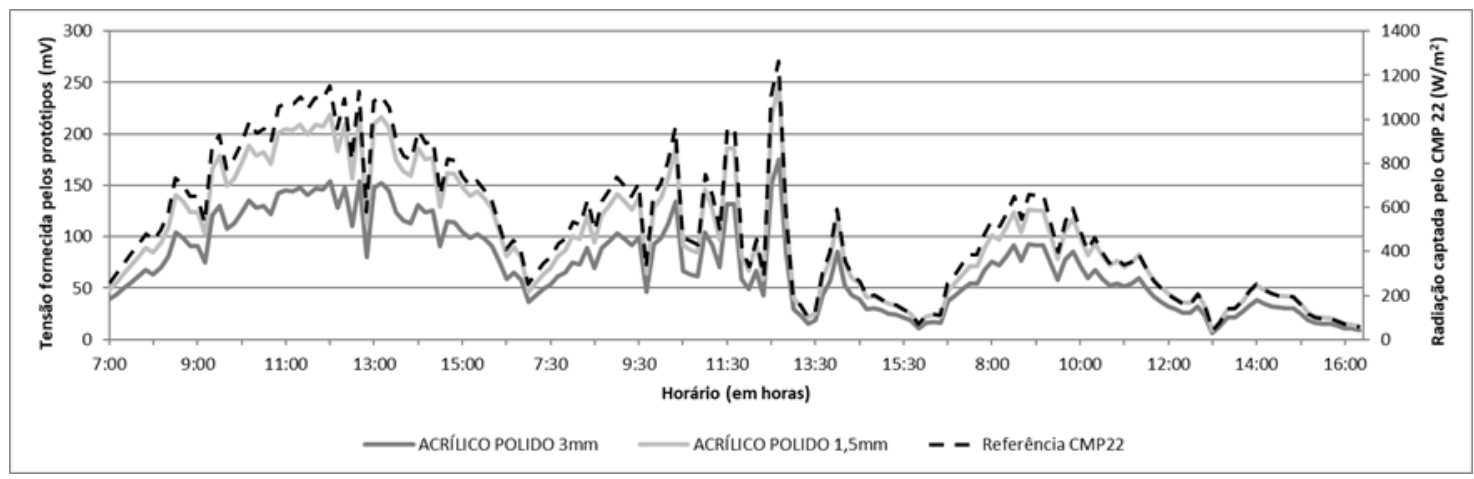

FiguRA 6. Resposta dos piranômetros com difusores de acrílico com espessuras de 1,5 e 3,0 mm comparativamente ao piranômetro de referência. (Autoria própria)

Ambos os difusores testados apresentaram resultados satisfatórios quando comparados com o piranômetro de referência. Se observa na Tabela 3, a partir da análise de regressão linear, os valores da sensibilidade e o coeficiente de determinação para os resultados de cada difusor, em relação à referência.

TABELA 3. Respostas dos piranômetros com difusores de acrílico com espessuras de 1,5 e 3,0 mm. (Autoria própria)

\begin{tabular}{|c|c|c|c|c|c|}
\hline \multirow{2}{*}{ Protótipo } & \multicolumn{2}{|c|}{ Difusor } & \multirow{2}{*}{ Sensibilidade } & Coeficiente de Variação & $\mathrm{R}^{2}$ \\
\cline { 2 - 3 } & Material & Espessura & & & \\
\hline 2 & Acrílico & $3 \mathrm{~mm}$ & $6,928 \mathrm{~W} \cdot \mathrm{m}^{-2} / \mathrm{mV}$ & $5,33 \%$ & 0,996 \\
\hline 3 & Acrílico & $1,5 \mathrm{~mm}$ & $5,043 \mathrm{~W} \cdot \mathrm{m}^{-2} / \mathrm{Mv}$ & $5,39 \%$ & 0,997 \\
\hline
\end{tabular}

De acordo com os resultados, o piranômetro com difusor de acrílico de 1,5 $\mathrm{mm}$ apresentou uma maior sensibilidade que o modelo com difusor de $3 \mathrm{~mm}$. Porém, o coeficiente de determinação $\left(\mathrm{R}^{2}\right)$ de ambos apresentaram valores muito próximos. Como ambos os protótipos têm elementos sensores muito semelhantes, a diferença de sensibilidade dos conjuntos indica que o sensor com o difusor de $3 \mathrm{~mm}$ recebe aproximadamente $27 \%$ menos radiações que o sensor com difusor de $1,5 \mathrm{~mm}$, o que deve contribuir para uma maior vida útil do primeiro. Assim, optou-se pela utilização do difusor de $3 \mathrm{~mm}$.

A terceira fase do experimento constituiu-se na análise do comportamento dos protótipos em relação ao acabamento superficial do difusor, quando foram testados modelos de superfície polida e opaca. O experimento foi realizado entre 7 e 11 de março de 2018, entre $8 \mathrm{~h} 00 \mathrm{~min}$ e próximo às $17 \mathrm{~h} 00 \mathrm{~min}$, intervalo de tempo em que o Sol se encontrava com um ângulo de elevação superior a $20^{\circ}$, e ocorreu com céu variando entre claro e nublado. Na Figura 7, pode ser observado o comportamento de dois piranômetros, um com difusor com superfície polida e outro com superfície opaca. 


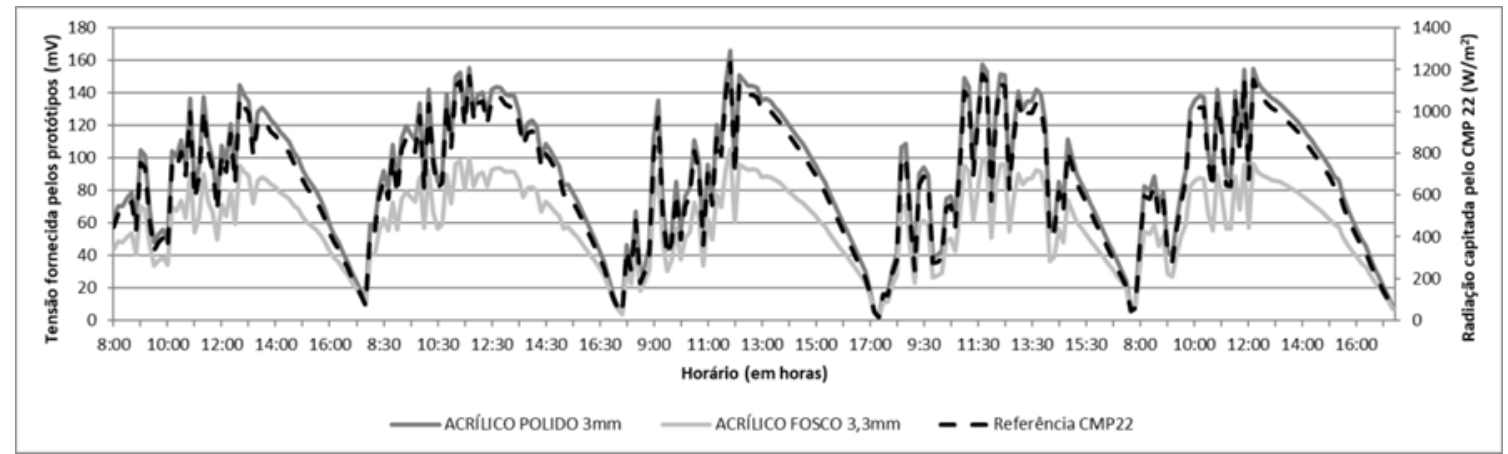

FIGURA 7. Resposta dos piranômetros com os difusores com acabamentos polido e fosco comparativamente ao piranômetro de referência (Autoria própria).

Devido à indisponibilidade de um segundo difusor de $3 \mathrm{~mm}$, foi utilizado um de 3,3 $\mathrm{mm}$, valor disponível mais próximo ao primeiro. Esta diferença foi desprezada na análise dos resultados. No Gráfico da Figura 7 , verifica-se que o modelo com difusor de acrílico fosco produziu menor tensão em relação ao polido e a Tabela 4 mostra os valores das regressões lineares das respostas de ambos os modelos.

TABELA 4. Resposta dos piranômetros com difusores de acrílico com superfícies polida e opaca (Autoria própria)

\begin{tabular}{|c|c|c|c|c|c|c|}
\hline \multirow{2}{*}{ Protótipo } & \multicolumn{2}{|c|}{ Difusor } & \multirow{2}{*}{ Espessura } & \multirow{2}{*}{ Sensibilidade } & \multirow{2}{*}{ Coeficiente de Variação } & \multirow{2}{*}{$\mathrm{R}^{2}$} \\
\hline & Material & Superfície & & & & \\
\hline 2 & Acrílico & Polida & $3 \mathrm{~mm}$ & $7,274 \mathrm{~W} \cdot \mathrm{m}^{-2} / \mathrm{mV}$ & $2,5 \%$ & 0,99 \\
\hline 3 & Acrílico & Opaco & $3,3 \mathrm{~mm}$ & $10,733 \mathrm{~W} \cdot \mathrm{m}^{-2} / \mathrm{mV}$ & $7,8 \%$ & 0,98 \\
\hline
\end{tabular}

Os dados da Tabela 4 mostram que o piranômetro com difusor de acrílico de superfície polida obteve melhor resultado, considerando a sensibilidade, coeficiente de variação e coeficiente de determinação. Portanto, o difusor com o material de acrílico leitoso polido, com espessura de $3 \mathrm{~mm}$ e acabamento polido foi selecionado para compor o piranômetro fotovoltaico de baixo custo proposto por este trabalho.

Por fim, foi determinado o erro relativo percentual absoluto do protótipo, escolhido como o modelo final, em relação ao piranômetro de referência, baseando-se nos resultados obtidos na última fase de testes. Na Figura 8 estão apresentados os valores dos erros relativos do protótipo em relação à irradiação incidente medida pelo piranômetro de referência.

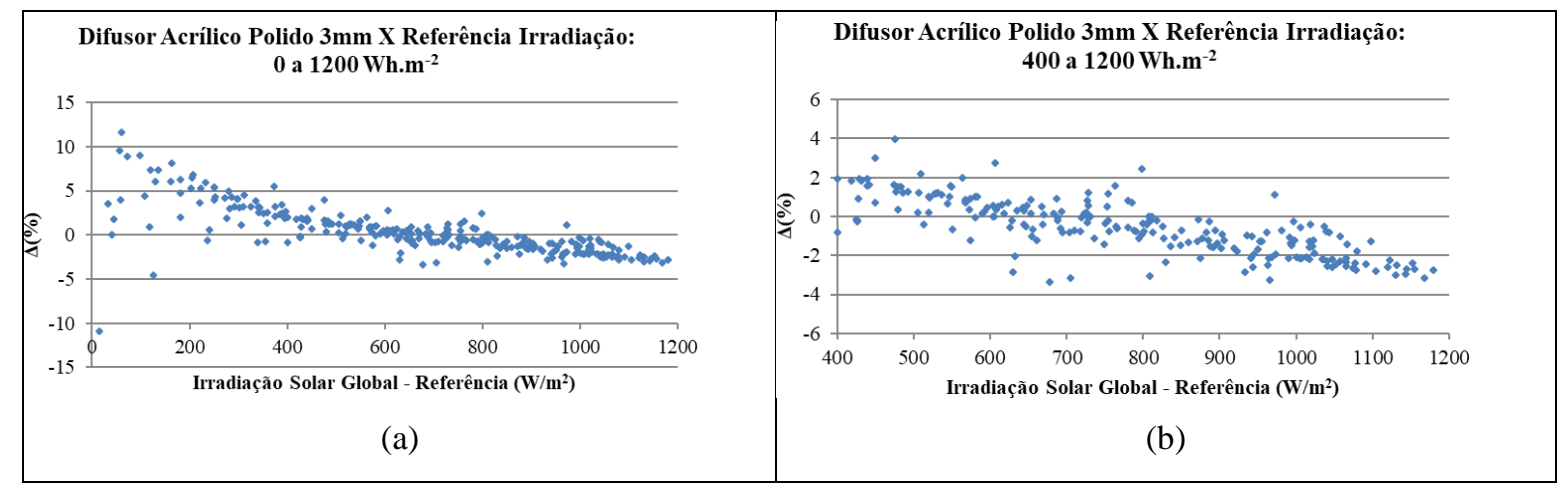

FIGURA 8. Erro relativo do piranômetro protótipo para irradiações até 1200 W.m² (a) e entre 400 e 1200 W.m 2 (b), comparativamente ao piranômetro de referência. (Autoria própria) 
Observam-se erros mais elevados para valores mais baixos de irradiação que, normalmente, correspondem aos horários de menores ângulos de elevação do Sol. O comportamento do erro em relação com a irradiação incidente é compatível com os resultados obtidos por outros dispositivos similares (Freira, 2008; Zanesco, 1991) e pode estar relacionado com o erro do cosseno, indicando que pode haver margem para melhorar o desempenho do protótipo, modificando-se a forma geométrica do difusor.

Nas medições de valores totais horários (Figura 9), o Erro Percentual Absoluto Médio foi mais elevado nos horários próximos ao nascer e ao pôr do sol, que correspondem aos mais baixos ângulos de elevação do Sol.

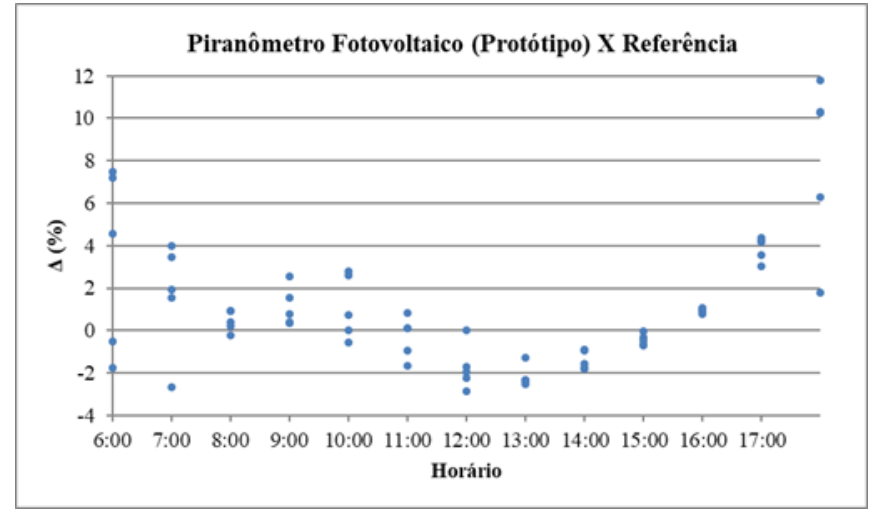

FIGURA 9. Erro relativo do piranômetro protótipo para medições totais horárias, comparativamente ao piranômetro de referência. (Autoria própria)

Os maiores valores do erro relativo percentual absoluto médio para totais horários foram registrados às $6 \mathrm{~h} 00 \mathrm{~min}$ e $18 \mathrm{~h} 00 \mathrm{~min}$, sendo 4,29\% e $8,10 \%$, respectivamente. Nesta escala, o erro médio mais baixo registrado foi de $0,433 \%$, às $15 \mathrm{~h} 00 \mathrm{~min}$, e o valor médio foi de $2,26 \%$. Para as medições de totais diários, o erro relativo percentual absoluto foi sempre inferior a $1 \%$, destacando-se o mínimo com 0,16\%, o máximo com 0,93\% e o valor médio de $0,5 \%$, conforme os resultados na Tabela 5 , o que indica um bom desempenho do protótipo.

TABELA 5. Resposta do piranômetro protótipo para medições totais diárias de irradiação, comparativamente ao piranômetro de referência. (Autoria própria)

\begin{tabular}{|c|c|c|c|}
\hline \multirow{2}{*}{ Data } & \multicolumn{2}{|c|}{ Irradiação Diária Total } & \multirow{2}{*}{ Diferença Percentual (\%) } \\
\cline { 2 - 3 } & KIPP\&ZONEN CMP22 & Piranômetro Fotovoltaico Protótipo & \\
\hline $07 / 03 / 2018$ & $6.421,36 \mathrm{kWh} \cdot \mathrm{m}^{-2}$ & $6.441,85 \mathrm{kWh} \cdot \mathrm{m}^{-2}$ & $0,32 \%$ \\
\hline $08 / 03 / 2018$ & $7.438,33 \mathrm{kWh} \cdot \mathrm{m}^{-2}$ & $7.368,85 \mathrm{kWh} \cdot \mathrm{m}^{-2}$ & $-0,93 \%$ \\
\hline $09 / 03 / 2018$ & $6.505,41 \mathrm{kWh} \cdot \mathrm{m}^{-2}$ & $6.487,24 \mathrm{kWh} \cdot \mathrm{m}^{-2}$ & $-0,28 \%$ \\
\hline $10 / 03 / 2018$ & $6.324,30 \mathrm{kWh} \cdot \mathrm{m}^{-2}$ & $6.314,41 \mathrm{kWh} \cdot \mathrm{m}^{-2}$ & $-0,16 \%$ \\
\hline $11 / 03 / 2018$ & $6.972,41 \mathrm{kWh} \cdot \mathrm{m}^{-2}$ & $6.916,70 \mathrm{kWh} \cdot \mathrm{m}^{-2}$ & $-0,80 \%$ \\
\hline
\end{tabular}

A partir dos dados obtidos pelo piranômetro de modelo final e considerando que o custo de construção foi inferior a $\mathrm{R} \$ 140,00$, pode-se afirmar que o protótipo apresentou um bom desempenho e possui baixo custo, em comparação com o piranômetro de referência, modelo KIPP\&ZONNEN CMP22, que é comercializado a um preço em torno de $£ 5.300,00$ (Libras) [8]. 


\section{CONCLUSÃO}

O piranômetro desenvolvido, elaborado com um pequeno módulo fotovoltaico, corpo de plástico de engenharia, e tendo como difusor uma placa de acrílico com formato cilíndrico e superfície polida, de fácil execução e baixo custo, apresenta um desempenho satisfatório para medições da irradiação total diária, com erro inferior a $1 \%$, em relação a um instrumento com sensor do tipo termopilha, de classe secundária pela classificação da norma ISO 9060, e alto custo.

Até mesmo medições instantâneas e de totais horários da irradiação podem ser realizadas com qualidade pelo protótipo proposto, desde que sejam observadas as limitações do instrumento para medidas para baixos ângulos de elevação do Sol.

Os resultados obtidos demonstram a viabilidade técnica e econômica do desenvolvimento de piranômetros fotovoltaicos de baixo custo, inferior a $\mathrm{R} \$ 140,00$, podendo estes quase que equiparar aos resultados do piranômetro de referência, KIPP\&ZONEN CMP22, o qual possui o mais alto padrão de classificação, conforme a ISO 9060, e um alto custo de aquisição.

\section{REFERÊNCIAS}

[1] Vilela, W.A. Estudo, desenvolvimento e caracterização de radiômetros para medidas de radiação solar. Tese de Doutorado, Instituto Nacional de Pesquisas Espaciais - INPE, São José dos Campos, SP, 09 de agosto de 2010.

[2] Freire, L. A. D. Desenvolvimento de um piranômetro fotovoltaico. Dissertação de Mestrado, Centro de Tecnologia e Geociências, Universidade Federal de Pernambuco - UFPE, Recife, PE, 03 de março de 2008.

[3] Zanesco, I. Análise e construção de um piranômetro fotovoltaico. Dissertação de Mestrado, Universidade Federal do Rio Grande do Sul - UFRGS, Porto Alegre, RS, 16 de julho de 1991.

[4] Fraidenraich, N.; Lyra, F. Energia Solar: fundamentos e tecnologia de conversão heliotérmica e fotovoltaica.; Editora: Editora Universitária da UFPE, Brasil, 1995; p. 471.

[5] Grupo de Trabalho de Energia Solar (GTES). Manual de engenharia de sistemas fotovoltaico. Rio de Janeiro, RJ: CRESESB, 2004.

[6] CAMPBELL SCIENTIFIC: when measurements matter. Disponível online: https://www.campbellsci.com/blog/pyranometers-need-to-know (acesso em 20/01/2018).

[7] KIPP \& ZONEN, Pyranometers. Disponível online: www.kippzonen.com (acesso em 15/01/2018).

[8] OMNI INSTRUMENTS. Disponível online: http://www.omniinstruments.co.uk/cmp22.html\#upsellproduct-div (acesso em 10/02/2018). 